\title{
Radiation-Induced Second Cancer Risk Estimates From Radionuclide Therapy
}

\author{
Bryan Bednarz ${ }^{1, *}$ and Abigail Besemer ${ }^{1}$ \\ ${ }^{1}$ Department of Medical Physics, University of Wisconsin, Madison, WI, 53705
}

\begin{abstract}
The use of radionuclide therapy in the clinical setting is expected to increase significantly over the next decade. There is an important need to understand the radiation-induced second cancer risk associated with these procedures. In this study the radiation-induced cancer risk in five radionuclide therapy patients was investigated. These patients underwent serial SPECT imaging scans following injection as part of a clinical trial testing the efficacy of a ${ }^{131}$ Iodine-labeled radiopharmaceutical. Using these datasets the committed absorbed doses to multiple sensitive structures were calculated using RAPID, which is a novel Monte Carlo-based 3D dosimetry platform developed for personalized dosimetry. The excess relative risk (ERR) for radiation-induced cancer in these structures was then derived from these dose estimates following the recommendations set forth in the BEIR VII report. The radiation-induced leukemia ERR was highest among all sites considered reaching a maximum value of approximately 4.5 . The radiation-induced cancer risk in the kidneys, liver and spleen ranged between 0.3 and 1.3. The lifetime attributable risks (LARs) were also calculated, which ranged from 30 to 1700 cancers per 100,000 persons and were highest for leukemia and the liver for both males and females followed by radiation-induced spleen and kidney cancer. The risks associated with radionuclide therapy are similar to the risk associated with external beam radiation therapy.
\end{abstract}

\section{Introduction}

Radionuclide therapy is a form of therapy involving the systemic administration of a radioactive compound in a patient. The primary basis for successful radionuclide therapy is the preferential uptake and retention of the radiopharmaceutical in the tumor without inducing acute or latent side effects in the patient. Radionuclide therapy has a proven track record in the clinical setting for treating a broad spectrum of solid and hematological cancers. The use of radionuclide therapy is expected to significantly increase in the next decade owing to the rapid development of a diverse set of novel radiopharmaceuticals.

While the risk of second cancer development from external beam radiation therapy (EBRT) has been investigated extensively [1-3], less is known about the radiation-induced cancer risk from radionuclide therapy. Because this risk is largely dependent on the absorbed dose and dose rate in the body one would expect sizeable differences in the risk predictions between these two treatments. The absorbed dose distribution resulting from radionuclide therapy is often heterogeneously distributed throughout the body of the patient unlike the absorbed dose from EBRT that falls off rapidly as a function of distance from the tumor. The absorbed dose from radionuclide therapy is delivered continuously over an extended period of time as governed by the radionuclides half-life, which is in stark contrast to EBRT where sizable doses are delivered to a tumor volume in multiple fractions. It is impossible to relate the risks between these treatment options without accounting for these aforementioned differences.

In order to provide adequate risk comparisons between EBRT and radionuclide therapy, new computational tools are needed. Currently, the only internal dosimetry software approved by the US Food and Drug Administration (FDA) is OLINDA/EXM (Organ Level Internal Dose Assessment for EXponential Modeling)[4]. OLINDA calculates absorbed doses using the formalism developed by the Medical Internal Radiation Dose (MIRD) committee of the Society of Nuclear Medicine [5]. OLINDA uses tabulated activity-to-dose scaling factors, or organ S-values (OSVs), generated based on standard phantoms [6]. Tumors are modeled separately as a unit-density sphere isolated in an infinite unit density medium. The major limitations of the OSV method implemented in OLINDA are that (1) phantoms that are not an accurate representation of each patient's unique geometry, (2) the radioactivity uptake and dose deposition are assumed to be uniformly distributed within each organ, and (3) the tumor not being modeled as part of the phantom leads to an underestimate of the normal tissue and tumor dose. Despite these limitations, OLINDA remains the only commercial internal dosimetry software utilized clinically because of its simplicity and ease of use.

The purpose of this work is to investigate radiation-induced second cancer risks from radionuclide therapy using an advanced Monte Carlo (MC) 3D dosimetry platform called RAPID (Radiopharmaceutical Assessment Platform for Internal Dosimetry).

\section{Methods and Materials}

\subsection{Dose Calculation Procedure}

The RAPID framework and workflow is demonstrated in Fig. 1. Serial 3D images (SPECT/CT) are acquired to map the agent's pharmacokinetics. Following acquisition, the images

*Corresponding author: bbednarz2@wisc.edu 
are coregistered, resampled, rescaled, corrected for partial volume effects, and masked. First, the PET/SPECT images are coregistered to the $\mathrm{CT}$ at each time point, a using normalized mutual information affine transformation with 12 degrees of freedom (3 rotational, 3 translational, 3 scaling, and 3 sheering). The SPECT data was then up-sampled to match the higher CT resolution. Given that Monte Carlo dosimetry relies both on accurate quantification of the activity distribution by the SPECT and the material composition/density by the $\mathrm{CT}$, each resampling option has both advantages and disadvantages. Resampling is performed using the Mitchell filter kernel that offers a good compromise between ringing and blurring artifacts.

The MC simulations in this work were done using the MC code Geant4 version 9.6 [7]. Geant4 is a versatile object-oriented simulation toolkit that allows for the modeling of complex geometries, radiation sources, and detectors. Geant 4 has been benchmarked for a variety of different medical physics applications [8-10]. The CT and the SPECT images are used in the Monte Carlo simulation to define the geometry and source distribution, respectively. The CT images define the material composition and mass density of the simulation geometry. For this work, the entire HU range was segmented into 27 materials based on the human tissue compositions defined by Schneider et al [11]. Each HU was assigned a unique mass density according to the conversion defined in Schneider et al [11]. The SPECT images were used to define the radionuclide activity in each voxel. The decay was $s$ simulated using the G4RadioactiveDecay module which allows the simulation of the decay of more than 2000 radioactive nuclei using the decay information from the ENSDF database [12]. The point of the radionuclide decay is sampled uniformly throughout each voxel.

The statistical relative error calculated on the voxel and ROI-level. Sufficient particles was simulated such that the average organ-level relative error was less than $1 \%$ and the average voxel-level relative error within the patient volume was less than $10 \%$. Note that the relative error only refers to the statistical precision of the Monte Carlo dose calculation and does not take in to account other sources of uncertainty such as the activity quantification, material/density quantification, coregistration, resampling, kinetic fitting, or ROI contouring.

To speed up the simulation time, the simulation jobs were parallelized into individual slices. The whole patient CT was used to define the patient geometry and calculate the dose deposition but each job only contains one slice of the SPECT defined source distribution. For example, a whole patient simulation would run 1 job containing 80 slices while a simulation parallelized into slices would run 80 jobs each containing 1 slice of the PET/SPECT defined activity distribution. The simulation efficiency of the slice parallelization method was compared with the whole patient method by calculating the absorbed dose rate distribution at a single time point based on a SPECT/CT image. It was shown that the slice parallelization simulations were 250-400 times faster and 3-5 times more efficient than the whole patient simulations. The image contained 80 slices with a matrix size of $512 \times 512$ voxels. All simulations were run on the UW Center for High Throughput Computing (CHTC) cluster.

Following the MC simulation at each time point the absorbed dose rate is co-registered to a single CT and resampled. The absorbed dose is calculated by time-integrating the absorbed dose rate in kinetic fitting module. Radiobiological dose metrics can also be calculated. The dosimetry output includes 3D dose distributions, biological equivalent dose (BED) distributions and equivalent uniform dose values in regions of interest.

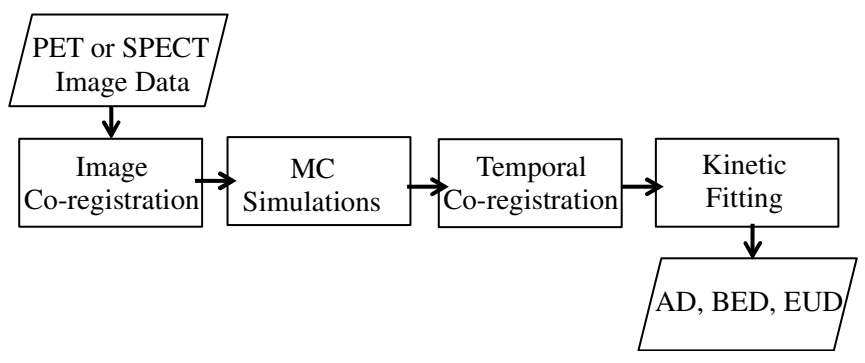

Figure 1. The RAPID framework and workflow.

For this investigation 5 patient datasets were analyzed from a Phase 1 dose escalation clinical trial investigating a 'theranostic/diapeutic' molecular agent, which can be radiolabeled with either ${ }^{124}$ I for PET imaging or ${ }^{131} \mathrm{I}$ for radiotherapy or SPECT/planar imaging [13]. Each patient underwent serial SPECT/CT ${ }^{131}$ I scans following treatment. Information about dosing and scan times are provided in Table 1. Following the workflow illustrated in Figure 1, the total committed absorbed dose distribution in each patient was determined. In addition committed absorbed doses were also calculated using the OLINDA for comparison.

Table 1. Injected ${ }^{131} \mathrm{I}$ dosage and scanning times for each patient considered in this investigation.

\begin{tabular}{|c|c|c|c|c|c|}
\hline \multirow[b]{2}{*}{ Patient } & \multirow{2}{*}{$\begin{array}{c}\text { Dose Group } \\
\left(\mathrm{mCi} / \mathrm{m}^{2}\right) / I \mathrm{nj} . \\
\text { Act. }(\mathrm{mCi})\end{array}$} & \multicolumn{4}{|c|}{ Scan Time Post Injection } \\
\hline & & Day 03 & Day 06 & Day 14 & Day 21 \\
\hline 1 & $25 / 48.6$ & 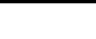 & 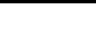 & 0 & 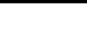 \\
\hline 2 & $37.5 / 84.5$ & - & ૫ & Q & प \\
\hline 3 & $37.5 / 64$ & - & Q & ( & Q \\
\hline 4 & $31.25 / 53.8$ & Q & Q & Q & Q \\
\hline 5 & $31.25 / 77.74$ & प & ( & ( & प \\
\hline
\end{tabular}

\subsection{Radiation Risk Estimation}

Second cancer risks to dose limiting organs from these treatments were calculated using the BEIR VII methodology [14]. Details of this methodology can be found in Chapter 12 of the BEIR VII report, but will be described below. The relationship between the risk of second cancer induction in 
exposed populations and the unexposed populations can be quantified by their difference known as the excess absolute risk (EAR) or their ratio known as the relative risk (RR). Typically, the excess relative risk (ERR) is used instead of $\mathrm{RR}$, where the ERR is equivalent to RR-1. That is the EAR and ERR are defined as the additive and multiplicative increase in incidence related to an exposure, respectively, where the incident rate is number of new cases of disease occurring in a specified amount of time among previously unaffected individuals. In this study, the age-dependent organ-specific ERRs were calculated for a variety of sites.

The BEIR VII report defines the ERR for solid tumors as a function of attained age (a) for patients over 30 as (Ref. 28):

$$
E R R=\rho(D) \cdot \beta_{s} \cdot(a / 60)^{\eta}
$$

where $e$ is the age at treatment, $\mathrm{D}$ is the total dose equivalent received and $\mathrm{s}$ is the gender of the patient. For solid tumor sites, the parameter $\rho(D)$ is a linear function of dose. The model parameters $\beta_{\mathrm{s}}$ and $\eta$ are provided for several organs in the BEIR VII report and are typically an order of magnitude different for the ERR.

The ERR for leukemia is defined by BEIR VII for patients over 30 as:

$$
E R R=\beta_{s} \cdot D(1+\theta D) \exp [\delta \log (t / 25]
$$

where $\mathrm{t}$ is the time since the treatment and the parameter $\theta$ indicates the degree of curvature of the linear quadratic model to represent the dose-response relationship for leukemia. The model parameters $\beta_{\mathrm{s}}$ and $\delta$ are provided for both ERR and EAR in BEIR VII. The absorbed dose to the bone marrow, which was determined within a small region of interest within the pelvic bone, was used to determine the ERR of inducing leukemia in these patients.

The lifetime attributable risk (LAR) is the probability that an exposed patient will develop a cancer during their lifetime. The BEIR VII report recommends taking a weighted average (log-scale) between the ERR and EAR to derive the LAR given as:

$$
\begin{gathered}
\operatorname{LAR}(D, e)=\left(\int_{a+L}^{100} M(D, e, a)_{E R R} \cdot S(a) / S(e) d a\right)^{0.7} \times \\
\left(\int_{a+L}^{100} M(D, e, a)_{E A R} \cdot S(a) / S(e) d a\right)^{0.3}
\end{gathered}
$$

where,

$$
\begin{aligned}
M(D, e, a)_{E R R} & =E R R(D, e, a) \cdot \lambda_{I}^{c} \text { and } \\
& M(D, e, a)_{E A R}=\operatorname{EAR}(D, e, a)
\end{aligned}
$$

where $S(a) / S(e)$ is the probability of a patient surviving from their exposure age (e) to their attained age (a) and were derived from life-span tables provided by Anderson and
DeTurk [15]. The recommended latent periods by BEIR VII of 5 years for solid cancers and 2 years for leukemia were used. The parameters $\lambda_{I}^{c}$ can be considered the baseline cancer risk for the exposed individual and are based on data provided in the SEER Cancer Statistics Review. The LAR coefficients derived from Eq. 1-4 and provided in BEIR VII were used for this work. The EAR values for the kidney and spleen were determined using the generic "Other solid cancer" coefficients set forth by BEIR VII. These coefficients were rescaled by a factor of 13 given that the "Other solid cancer" category combines risk for 13 remainder organs. Organ-specific coefficients were used for all remaining organs. The risks derived for leukemia assumed that the age of the exposure was 30 years. All other solid cancer risks assumed the ERR was independent of the age at exposure following recommendations from the BEIR VII report. The risk coefficients were rescaled by a factor of 1.5 to eliminate the dose and dose rate effectiveness factor (DDREF) used by BEIR committee to adjust for low dose rate exposures. This is based on the assumption that ${ }^{131} \mathrm{I}$ therapy can be considered a high dose and dose rate therapy due to biological clearance of CLR1404. A more appropriate approach, albeit out of the scope of this work, would be to determine DDREF with a value between 1 and 1.5 specific to this type of therapy.

\section{Results}

Mean absorbed doses to the kidneys, spleen, liver, and bone marrow were calculated using RAPID from these treatments are provided in Figure 2. Also included are mean doses calculated by OLINDA. As seen in Figure 2 the mean absorbed dose calculated by RAPID ranged between 1.66 3.44 Gy. There are clear differences between absorbed doses by RAPID using patient-specific MC simulations and OLINDA where these differences can be attributed to the aforementioned weaknesses of the OLINDA software. RAPID calculates the committed absorbed dose in each voxel using patient-specific images as illustrated in the dose color washes provided in Figure 3 for Patient 2. One would expect similar variations in the ERR values calculated for these patients using absorbed dose values from these two sources.

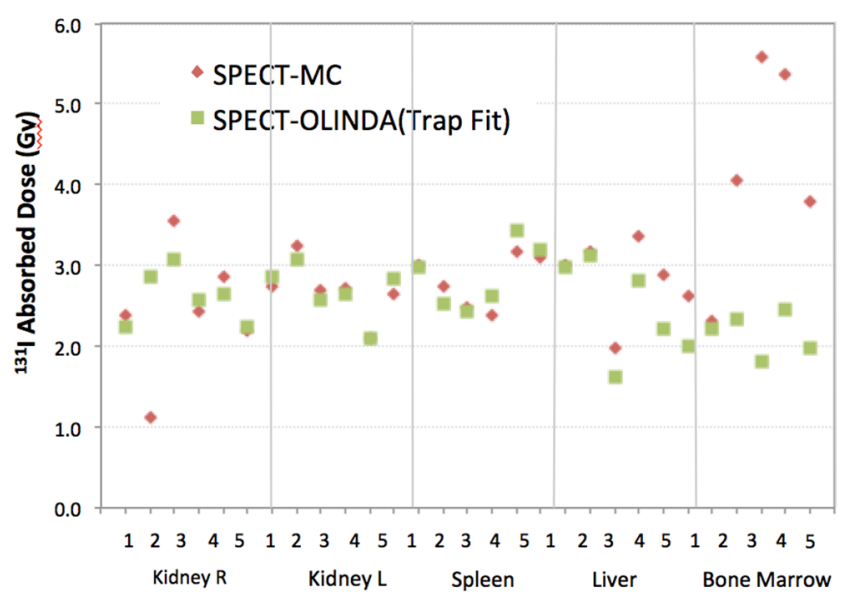


Figure 2. Mean committed absorbed doses in the kidneys, spleen, liver, and bone marrow for each patient calculated with RAPID and OLINDA.
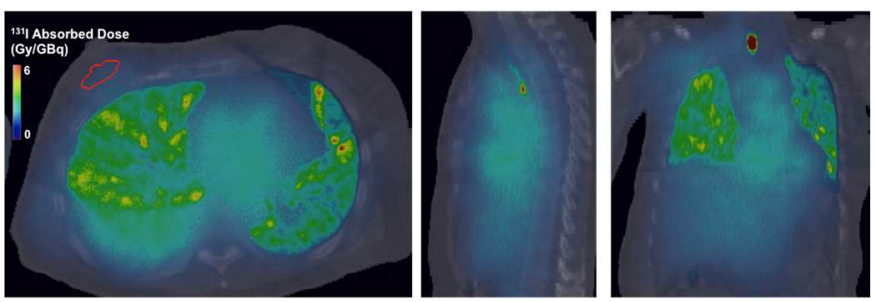

Figure 3. Absorbed dose colorwashes normalized per injected activity shown in the transverse, sagittal and corona 1 plane for Patient 2.

Figure 4 (a-e) provides sex-averaged ERR values for radiation-induced cancers in the kidney, spleen, liver, and blood. The highest ERR values are associated with leukemia and the lung whereas the liver, kidneys and spleen all had lower ERR values. Relatively large patient risk variations are seen within different cancer groupings.

Table 2 provides the LAR for inducing cancer in the kidney, spleen, liver and blood as a function of age derived using the ERR values provided above and the EAR values (data not shown). LARs for both males and females are included.

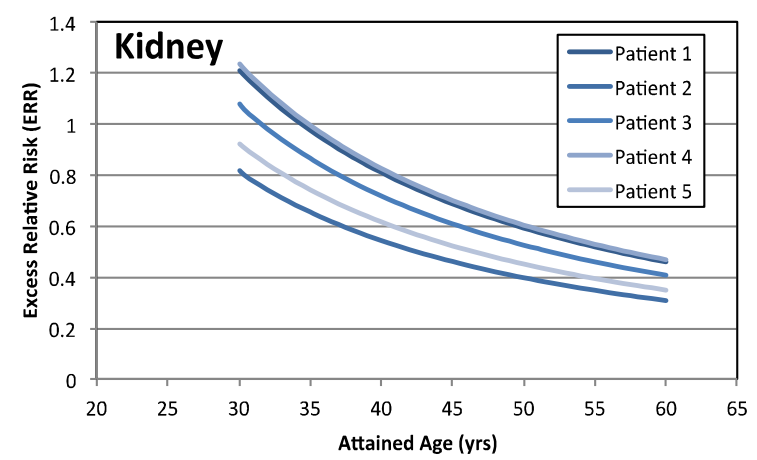

(a.)

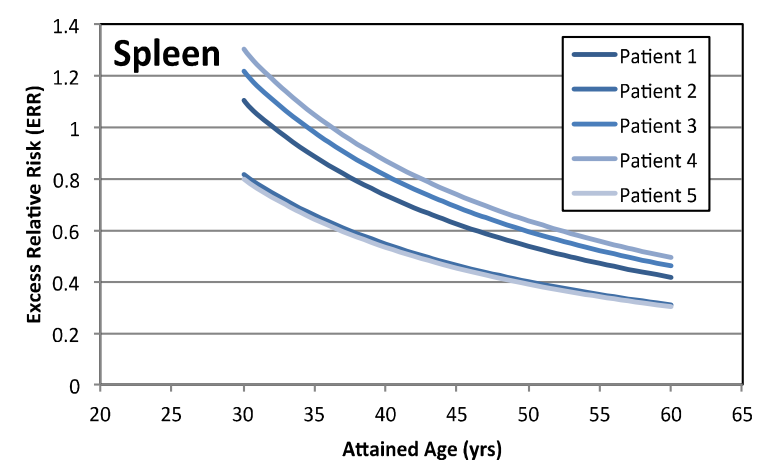

(b.)

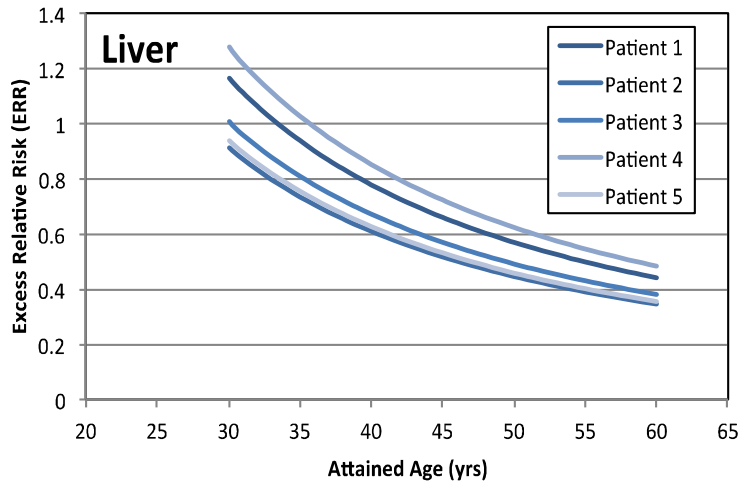

(c.)

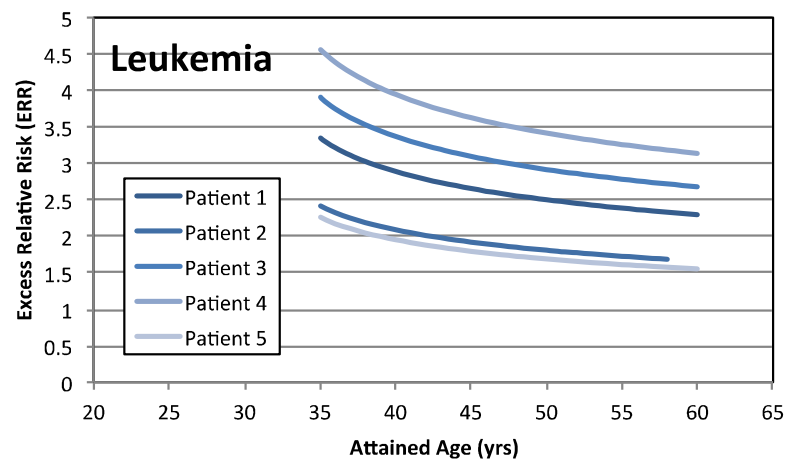

(d.)

Figure 4. Sex-averaged ERR values for (1.) kidney, (b.) spleen, (c.) liver, and (d.) leukemia for the five patients considered in this study.

\section{Discussion and Conclusion}

This work investigated the risk of radiation-induced second cancers in radionuclide therapy patients. The committed absorbed dose to multiple sensitive structures was calculated using RAPID. The sex-averaged ERR and LAR of developing a radiation-induced cancer in these structures was then derived following the recommendations set forth in the BEIR VII report [7].

Our results indicate that the sex-averaged ERR in these patients range from 0.5 to 4.5 depending on the organ site, absorbed dose to the site, and obtained age of the patient following the exposure. The LARs associated with these exposures ranged roughly from 30 to 1700 cancers per 100,000 persons. The LAR risks were highest for radiation-induced leukemia risks for both males and females followed by radiation-induced liver cancer. This likely due to the relatively slow clearance of CLR1404 from the bloodstream.

There has been heightened interest and concern about radiation-induced cancer risk in external beam radiation therapy in recent years. The initial results from this work indicate that the risk of radiation-induced second cancers from radionuclide therapy are similar to those predicted for external beam radiation therapy [16]. However, the risk of radiation-induced leukemia is potentially much 
higher given the differences in dose distributions involved. This is supported by several clinical studies on radiation-induced leukemia in patients treated with ${ }^{131} \mathrm{I}$ for thyroid cancer [17]. Given these findings, it is imperative that more attention be afforded to investigating the cancer risks associated with radionuclide therapy.

\section{Acknowledgments}

We would like to thank Dr. Joseph Grudzinski and Mr. Alex Newell for help with data collection and analysis. We would like to thank the UW Center for High Throughput Computing (CHTC) for the use of their cluster and their computational support.

\section{References}

[1] X. G. Xu, B. Bednarz, and H. Paganetti, Phys. Med. Biol. 53, R193R241 (2008)

[2] E. J. Hall and C. S. Wuu, Int. J. Radiat. Oncol., Biol., Phys. 56, 83-88 (2003)

[3] M. Tubiana Radiother. Oncol. 91, 4-15 (2009)

[4] M.G. Stabin, R.B. Sparks, E. Crowe, J Nucl Med, 46(6), 1023-1027 (2005)

[5] W.E. Bolch, K.F. Eckerman, G. Sgouros, S.R. Thomas Journal of Nuclear Medicine 50, 477-484 (2009).

[6] M.G. Stabin, E. Watson, M. Cristy, J. Ryman, K. Eckerman, J. Davis, D. Marshall, M. Gehlen ORNL Report (1995)

M. Cristy, K. Eckerman, ORNL/TM-8381 6 (1987)

[7] Agnostinelli, Nuclear Instruments and Methods in Physics Research Section A: Accelerators, Spectrometers, Detectors and Associated Equipment 506, 250 - 303 (2003).

[8] B.A. Faddegon, I. Kawrakow, Y. Kubyshin, J. Perl, J. Sempau, L. Urban, Phys. Med. Biol 542009).

[9] T. Aso, A. Kimura, S. Tanaka, H. Yoshida, N. Kanematsu, T. Sasaki, T. Akagi, Nuclear Science, IEEE Transactions on 52, 896-901 (2005).

[10] C. Thiam, V. Breton, D. Donnarieix, B. Habib, L. Maigne, Phys. Med. Biol. 53, 3039 (2008).

[11] W. Schneider, T. Bortfeld, W. Schlegel Phys. Med. Biol. 53, 459 (2000)

[12] S. Hauf, M. Kuster, M. Batic, Z.W. Bell, D.H. Hoffman, P.M. Lang, et al Nuclear Science IEEE Trans. 60, 2966-2983 (2013)

[13] J. Grudzinski, B. titz, K. Kozak, W. Clarke, et al et al PLOS one 9 (11) 1-11 (2014)

[14] BEIR, Health Risks from Exposure to Low Levels of Ionizing Radiation: BEIR VII, Phase 2. National Academy of Science, Washington, DC, (2006)

[15] R.N. Anderson and P.B. DeTurk, National Vital Statistics Report, 50(6), 1-12 (2002)

[16] Shuryak I et al. J Natl Cancer Inst. 98(24) 1794-1806 (2006)

[17] Fard-Esfahani A, Emamu-Ardekani A, Fallahi B, et al Nucl Med Commun 35(8), 808-817 (2014)

Table 2: LAR as a function of cancer site and age at exposure (yr) for the five patients considered in this study. LAR (cases per 100,000 persons)

\begin{tabular}{|c|c|c|c|c|c|c|c|c|c|c|c|c|}
\hline & \multicolumn{12}{|c|}{ Age at exposure (yr) } \\
\hline & \multicolumn{2}{|r|}{30} & \multicolumn{2}{|c|}{40} & \multicolumn{2}{|c|}{50} & \multicolumn{2}{|c|}{60} & \multicolumn{2}{|c|}{70} & \multicolumn{2}{|r|}{80} \\
\hline \multicolumn{13}{|l|}{ Organ } \\
\hline Patient 1 & Male & Female & Male & Female & Male & Female & Male & Female & Male & Female & Male & Female \\
\hline Kidney & 291 & 304 & 253 & 266 & 206 & 218 & 144 & 160 & 84 & 100 & 34 & 44 \\
\hline Liver & 455 & 207 & 434 & 207 & 393 & 186 & 290 & 145 & 165 & 103 & 62 & 41 \\
\hline Spleen & 265 & 277 & 230 & 242 & 187 & 198 & 131 & 146 & 76 & 91 & 31 & 40 \\
\hline Leukemia & 1353 & 1015 & 1353 & 999 & 1353 & 999 & 1321 & 918 & 1176 & 822 & 773 & 596 \\
\hline \multicolumn{13}{|l|}{ Patient 2} \\
\hline Kidney & 195 & 204 & 170 & 179 & 138 & 146 & 97 & 108 & 56 & 67 & 23 & 30 \\
\hline Liver & 356 & 162 & 340 & 162 & 307 & 146 & 226 & 113 & 129 & 81 & 49 & 32 \\
\hline Spleen & 197 & 206 & 171 & 180 & 139 & 147 & 97 & 108 & 57 & 68 & 23 & 30 \\
\hline Leukemia & 1090 & 817 & 1090 & 804 & 1090 & 804 & 1064 & 739 & 947 & 662 & 623 & 480 \\
\hline \multicolumn{13}{|l|}{ Patient 3} \\
\hline Kidney & 258 & 269 & 224 & 236 & 182 & 193 & 128 & 142 & 74 & 89 & 30 & 39 \\
\hline Liver & 356 & 179 & 340 & 179 & 307 & 161 & 226 & 125 & 129 & 89 & 49 & 36 \\
\hline Spleen & 293 & 307 & 255 & 268 & 207 & 219 & 145 & 161 & 84 & 101 & 34 & 44 \\
\hline Leukemia & 1501 & 1125 & 1501 & 1108 & 1501 & 1108 & 1465 & 1018 & 1304 & 911 & 858 & 661 \\
\hline \multicolumn{13}{|l|}{ Patient 4} \\
\hline Kidney & 297 & 310 & 258 & 271 & 210 & 222 & 147 & 163 & 85 & 102 & 34 & 45 \\
\hline Liver & 498 & 227 & 476 & 227 & 431 & 204 & 317 & 159 & 181 & 113 & 68 & 45 \\
\hline Spleen & 312 & 326 & 271 & 285 & 221 & 233 & 154 & 172 & 90 & 107 & 36 & 47 \\
\hline Leukemia & 1668 & 1251 & 1668 & 1231 & 1668 & 1231 & 1628 & 1132 & 1450 & 1013 & 953 & 735 \\
\hline \multicolumn{13}{|l|}{ Patient 5} \\
\hline Kidney & 221 & 232 & 192 & 202 & 157 & 166 & 110 & 122 & 64 & 76 & 26 & 34 \\
\hline Liver & 365 & 166 & 348 & 166 & 315 & 149 & 232 & 116 & 133 & 83 & 50 & 33 \\
\hline $\begin{array}{l}\text { Spleen } \\
\text { Leukemia }\end{array}$ & $\begin{array}{r}193 \\
1031\end{array}$ & $\begin{array}{l}202 \\
773\end{array}$ & $\begin{array}{r}167 \\
1031\end{array}$ & $\begin{array}{l}176 \\
761\end{array}$ & $\begin{array}{r}136 \\
1031\end{array}$ & $\begin{array}{l}144 \\
761\end{array}$ & $\begin{array}{r}95 \\
1006\end{array}$ & $\begin{array}{l}106 \\
700\end{array}$ & $\begin{array}{r}56 \\
896\end{array}$ & $\begin{array}{r}66 \\
626\end{array}$ & $\begin{array}{r}22 \\
589\end{array}$ & $\begin{array}{r}29 \\
454\end{array}$ \\
\hline
\end{tabular}

